\title{
In-Vitro Validation of a Novel Model-Based Approach to the Measurement of Arterial Blood Flow Waveforms from Dynamic Digital X-ray Images
}

\author{
Kawal Rhode ${ }^{1}$, Gareth Ennew ${ }^{1}$, Tryphon Lambrou ${ }^{1}$, Alexander Seifalian ${ }^{2}$, and \\ David Hawkes ${ }^{1}$ \\ ${ }^{1}$ Division of Radiological Sciences and Medical Engineering, Guy's, King's and St. Thomas' \\ Hospitals Medical School, Guy's Hospital, London, U.K., SE1 9RT \\ ${ }^{2}$ University Department of Surgery, Royal Free and University College Medical School, \\ The Royal Free Hospital, Pond Street, London, U.K., NW3 2QG
}

\begin{abstract}
We have developed a blood flow waveform shape model using principal component analysis (PCA) and applied this to our existing concentration-distance curve matching technique for the extraction of flow waveforms from dynamic digital $x$-ray images. The aim of the study was to validate the system using a moving-vessel flow phantom. Instantaneous recording of flow from an electromagnetic flow meter (EMF) provided the "gold standard" measurement. A model waveform was constructed from 256 previously recorded waveforms from the EMF using PCA. Flow waveforms were extracted from parametric images derived from dynamic $\mathrm{x}$-ray data by finding the parameters of the shape model that minimized the mean value of our cost function. The computed waveforms were compared to the EMF recordings. The model-based approach produced narrower limits of agreement with the EMF data than our previously developed algorithms and, in the presence of increasing noise in the parametric images, it out-performed the other algorithms.
\end{abstract}

\section{Introduction}

The algorithms ${ }^{(1)}$ that have been previously reported for the measurement of blood flow in arteries from dynamic digital x-ray images have not fully taken into account the nature of the blood flow in the target artery. At most, investigators have made assumptions about the different velocity profiles that exist radially across an arterial lumen. With the advent of the use of Doppler ultrasound to monitor blood flow in arteries, it became apparent that the shape and content of the Doppler sonogram was characteristic of the artery being investigated and the disease state of this artery. Investigators have attempted to characterize sonograms with a view to distinguish between healthy and diseased states ${ }^{(2-7)}$. Our method ${ }^{(8)}$ of velocity waveform measurement necessarily over samples the waveform yet is severely noise-limited. The lower bound of the sampling rate is determined by the distance traveled by the blood per sampling interval in relation to the sampled vessel length. Therefore, we hypothesized that the a priori knowledge of the nature of the blood flow through a target artery may be used to improve the measurement of blood flow through that W. Niessen and M. Viergever (Eds.): MICCAI 2001, LNCS 2208, pp. 291-300, 2001.

(C) Springer-Verlag Berlin Heidelberg 2001 
artery using dynamic digital x-ray images. This hypothesis was tested by constructing a blood flow waveform shape model from many waveforms collected by electromagnetic flow meter recordings using a blood flow test circuit under varying conditions of flow. This shape model was then used to constrain the measurement of blood flow in a blood flow circuit using the concentration-distance curve-matching algorithm previously developed ${ }^{(8)}$.

\section{Method}

\subsection{Physiological Blood Flow Circuit}

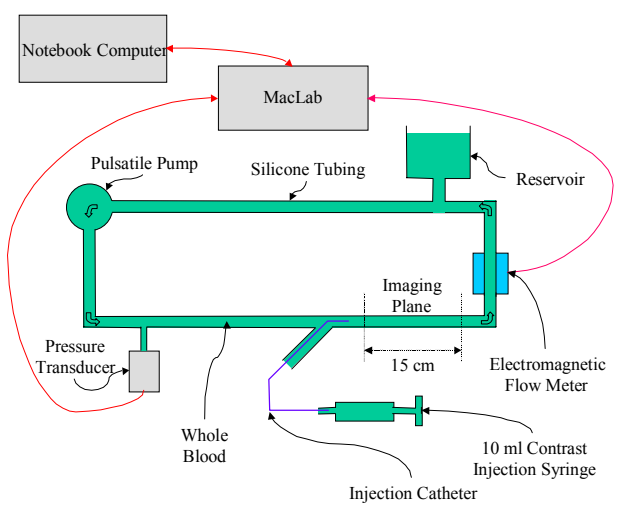

Fig. 1. Physiological blood flow circuit.

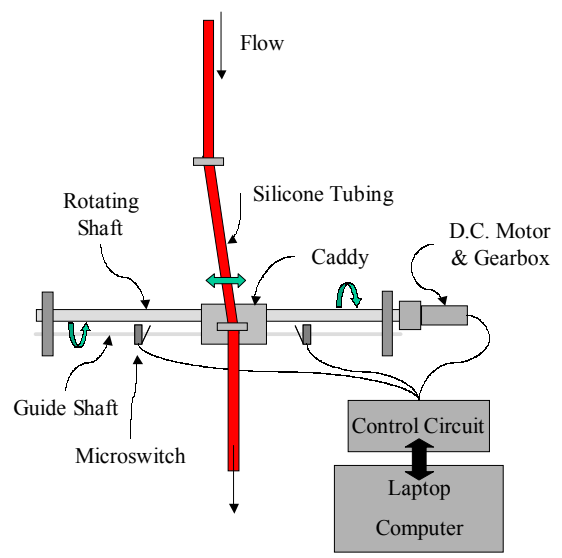

Fig. 2. Programmable vessel manipulator.

Figure 1 shows a schematic of the physiological blood flow circuit used to simulate pulsatile blood flow in the human circulation. A $15 \mathrm{~cm}$ section of silicone tubing was used to simulate a blood vessel. Date-expired whole blood was obtained and used as the circulating fluid. Pulsatile flow was generated using a pulsatile syringe pump (Pulsatile Blood Pump 1405, Harvard Apparatus). This allowed adjustment of mean flow rate by two means: (1) by altering the pumping frequency; (2) by altering the stroke volume. A $6 \mathrm{~mm}$ calibre electromagnetic flow meter (Electromagnetic Blood Flow Sensor / Electromagnetic Blood Flow and Velocity Meter, Skalar) was placed downstream from the simulated blood vessel. This provided outputs of instantaneous and mean flow rate. These were recorded using an analogue recording system (MacLab 8s, AD Instruments) that was interfaced to a Macintosh notebook. Our x-ray technique was validated by correlating $\mathrm{x}$-ray measurements with those made independently by the EMF. The pressure in the circuit was monitored using a pressure transducer connected to the MacLab. Physiological pressures were maintained by varying the height of the fluid reservoir. A 4-F catheter was inserted just upstream from the blood vessel to allow injection of iodine-based contrast medium using a 10 $\mathrm{ml}$ syringe. Figure 2 shows the programmable vessel manipulator that simulated vessel motion such as that seen in the coronary arteries during the cardiac cycle. This consisted of a geared d.c. electric motor driving a caddy on a linear axis. The motor 
speed was controlled using a pulse-width modulation electronic circuit. The motion of the manipulator could be programmed using a laptop computer that was interfaced to the control circuit. The simulated vessel was mounted with one end on the moving caddy and with the other end fixed. The motion of the manipulator was synchronized to the pulsatile pump.

\subsection{Model Waveform Construction}

In order to form a model of the blood flow waveforms that are produced by our blood flow circuit, we collected sample waveforms from the EMF under varying conditions of flow. The parameters altered were: (1) the stroke volume of the pump; (2) the pumping frequency; (3) the mean pressure; (4) the output impedance; (5) the vessel size; and (6) the rate of contrast medium injection. For each flow condition approximately 10 seconds of the flow signal was captured that included a 3 second period of contrast medium injection. Figure 3 shows a typical blood flow signal recorded from the EMF. For each recording the individual cycles were isolated. This was performed automatically by software that identified the systolic foot of each cycle in the recording by searching for 5 consecutive positive gradients that exceeded a predefined threshold. The foot was then marked as the first point in this series of gradients. The cycles isolated during the contrast injection phase were used as input to the principal component analysis.

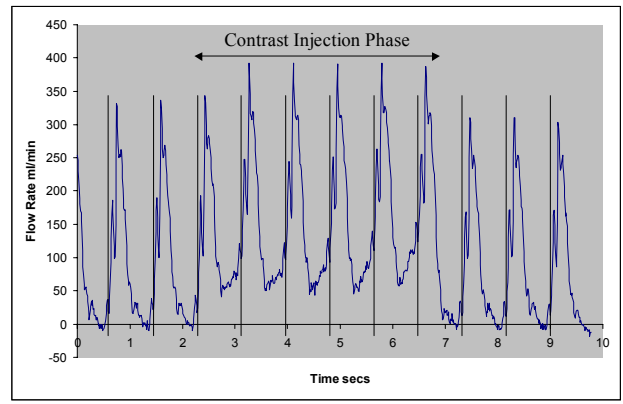

Fig. 3. An example of a recording from the electromagnetic flow meter showing identification of the systolic feet.

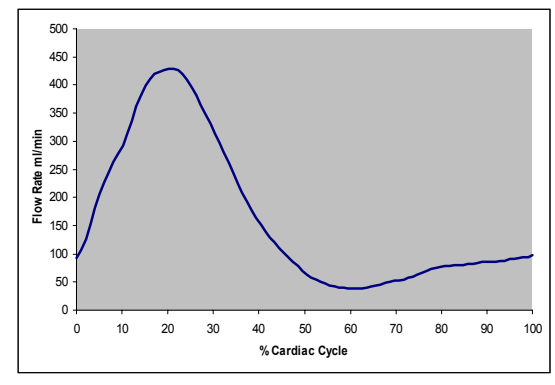

Fig. 4. Mean model waveform shape.

Each cycle was normalized over time by resampling by linear interpolation using $S=100$ sample points. The mean flow rates ranged from $43 \mathrm{ml} / \mathrm{min}$ to $300 \mathrm{ml} / \mathrm{min}$. The instantaneous flow rates ranged from $-175 \mathrm{ml} / \mathrm{min}$ (reverse flow) to $901 \mathrm{ml} / \mathrm{min}$. In total $N=256$ input waveforms were used. Each input waveform $\mathbf{P}_{i}$ consists of $S$ sample points $\left\{q_{i 1}, q_{i 2}, q_{i 3}, \ldots . ., q_{i S}\right\}$. The input waveforms were averaged to produce a mean waveform shape (figure 4 )

$$
\overline{\mathbf{P}}=\frac{1}{N} \sum_{i=1}^{i=N} \mathbf{P}_{i}
$$


The input waveforms were then transformed by subtracting the mean waveform shape

$$
\mathbf{d} \mathbf{P}_{i}=\mathbf{P}_{i}-\overline{\mathbf{P}}=\left\{d q_{i 1}, d q_{i 2}, d q_{i 3}, \ldots \ldots, d q_{i S}\right\} \text { for } 1 \leq i \leq N .
$$

The $S \times S$ covariance matrix $\mathbf{C}$ was calculated

$$
C_{i j}=\frac{1}{N} \sum_{k=1}^{k=N} d q_{k i} \times d q_{k j} \text { for } 1 \leq i, j \leq S .
$$

Eigen analysis of this real symmetric covariance matrix was performed. This yielded $S$ normalized eigenvectors $\mathbf{e}_{j}$ with corresponding eigenvalues $\lambda_{j}(1 \leq j \leq S)$. The eigenvectors were sorted in order of decreasing eigenvalue. The eigenvectors form a basis vector set for the transformed input waveforms $\mathbf{d P}_{i}$. Each eigenvector accounts for one mode of variation of the mean waveform shape $\overline{\mathbf{P}}$ with variance equal to the corresponding eigenvalue. Any of the input waveforms $\mathbf{P}_{i}$ can now be expressed as the sum of the mean waveform shape and a linear combination of the eigenvectors

$$
\mathbf{P}_{i}=\overline{\mathbf{P}}+\sum_{j=1}^{j=S} w_{i j} \mathbf{e}_{j} \text { for } 1 \leq i \leq N
$$

where $w_{i j}$ is the weighting factor for eigenvector $\mathbf{e}_{j}$ for waveform $i$. The percentage variation $v \%_{j}$ attributed to eigenvector $\mathbf{e}_{j}$ can be calculated as

$$
v \%_{j}=\frac{\lambda_{j}}{\sum_{i=1}^{i=S} \lambda_{i}} \times 100 \text { for } 1 \leq j \leq S
$$

where $\lambda_{j}$ is the eigenvalue associated with eigenvector $\mathbf{e}_{j}$.

Figure 5 shows cumulative percentage variation contributed by the first 20 eigenvectors. It was found that the first 10 eigenvectors are needed to explain $99.7 \%$ of the total variation in the waveform shape. Figures 6 shows the effect of the first eigenvector on the mean shape. It is possible with PCA to see that a particular eigenvector affects a particular aspect of the shape, however, this is not always the case. From the results it can be noted that: (1) the first eigenvector primarily affects the amplitude of the systolic peak; (2) the second eigenvector affects the placement of the systolic peak; and (3) the third eigenvector affects the gradient of the falling edge of the systolic peak. These observations are generalized and each eigenvector has more than just one effect on the waveform shape. The less significant eigenvectors have very little effect on the waveform shape. Therefore, it is possible to reconstruct each of the input waveforms by finding only the weighting factors for the first few eigenvectors. The percentage noise in the input waveform signals was estimated to be $0.5 \%$. Therefore, it was decided to use the first 10 eigenvectors to characterize the waveform shape since this number explained more than $99.5 \%$ of the total variation. 


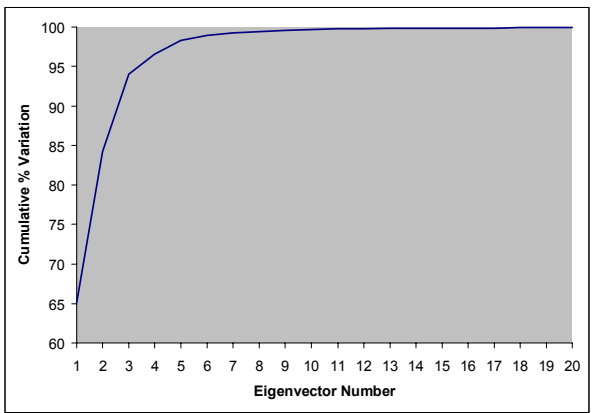

Fig. 5. Percentage cumulative variation accounted for by the first 20 eigenvectors.

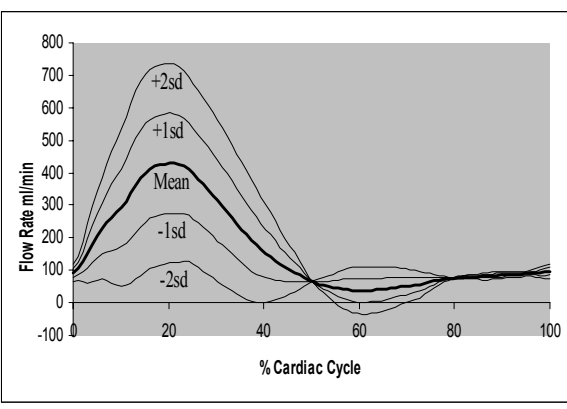

Fig. 6. Effect on the mean waveform shape of adding or subtracting 1 and 2 standard deviations of the first eigenvector.

\subsection{X-ray Image Acquisition}

For the x-ray experiment, a $6 \mathrm{~mm}$ internal diameter vessel was used. The vessel manipulator gave a maximum vessel speed of $29 \mathrm{~mm} / \mathrm{sec}$ and the range of vessel travel was $40 \mathrm{~mm}$. The frequency of motion and of the pump were set to 43 per min. X-ray images were acquired using an Advantx DX (GE Medical Systems) x-ray system. The images were transferred to a PC in real-time by digitisation of the PAL composite video signal normally available for recording of images to a video tape recorder. The x-ray system was set for $5 \mathrm{~ms}$ pulsed mode at 25 frames per second. The image acquisition was carried out by a PC workstation using a Pulsar frame capture card (Matrox Imaging). Images were digitised on a 512 x 512 pixel matrix at 8 bits grey depth and image grey level was proportional to the logarithm of the x-ray image brightness. The $\mathrm{x}$-ray tube voltage was $85 \mathrm{kV}$. The simulated blood vessel was placed in the isocentre of the x-ray system. The stroke volume of the pulsatile pump was adjusted to achieve the required mean flow rate and the system was allowed to reach a steady state. Initially a short sequence of images covering at least 1 cycle was acquired prior to the injection of iodine contrast medium, the pre-contrast sequence. This was followed by the injection of the contrast medium via a catheter just upstream from the segment under investigation. The rate of injection was varied depending on the mean flow rate from $2 \mathrm{ml} / \mathrm{sec}$ to $3 \mathrm{ml} / \mathrm{sec}$ over 3 seconds. A 3 second acquisition of 75 images was performed during the contrast injection phase. This sequence is known as the post-contrast sequence. Biplanar acquisition was used to determine the three-dimensional course of the blood vessel. For each flow rate, one set of images was acquired with the $\mathrm{x}$-ray c-arm at the $\mathrm{L} 40^{\circ}$ position and one at $\mathrm{R} 40^{\circ}$. To calibrate the geometry of the x-ray system, a purpose designed $60 \mathrm{~mm}$ Perspex cube with 14 radio-opaque markers at known positions was used. Images of the cube were acquired under the same two projections used for imaging the blood vessel.

To enable quantification of the iodine in the blood vessel, image subtraction was carried out. The technique of phase-match subtraction was used. The images in the pre-contrast sequence were matched in postion of the cardiac cycle to the images in the post-contrast sequence using the extremes of vessel motion for synchronisation. 
The pre-contrast images were then subtracted from the post-contrast images to leave just the iodine signal.

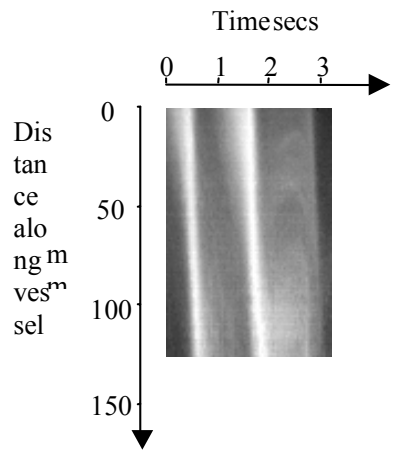

Fig. 7. Example of a parametric image produced from the angiographic data.

The subtracted x-ray images from the biplanar acquisition for each experiment were then analyzed on a computer workstation using a software package developed by our group (SARA - System for Angiographic Reconstruction and Analysis) ${ }^{(9)}$. This produced a parametric image from the angiographic data in which the image grey level represents contrast material concentration as a function of time and distance along a vessel segment (figure 7). In total 10 experiments were performed for different flow rates. This yielded 19 complete cardiac cycles for comparison with the EMF recordings.

\subsection{Extraction of Blood Flow Waveforms from Parametric Images}

The concentration of contrast medium along an arterial vessel segment can be expressed as a function of distance along the vessel and of time, $\mathbf{C}(x, t)$. The distance $x$ is measured in millimeters and $0 \leq x \leq N$. The segment length is then $(N+1) \mathrm{mm}$. The time $t$ is measured in frames and $0 \leq t \leq T$. This gives rise to a series of concentration-distance curves $\mathbf{C}(x, 0), \mathbf{C}(x, 1), \mathbf{C}(x, 2), \ldots \ldots, \mathbf{C}(x, T)$ where $T$ is the last frame in the series. If curve $\mathbf{C}(x, t)$ is shifted by $s \mathrm{~mm}$ with respect to curve $\mathbf{C}(x, t+1)$, the cost function $\mathbf{\Psi}(s, t)$ is calculated as

$$
\begin{aligned}
& \Psi(s, t)=\frac{1}{N-s+1} \sum_{x=0}^{x=N-s}(C(x, t)-C(x+s, t+1))^{2} \text { if } s \geq 0 \\
& \Psi(s, t)=\frac{1}{N-s+1} \sum_{x=0}^{x=N-s}(C(x-s, t)-C(x, t+1))^{2} \text { if } s<0 .
\end{aligned}
$$

This cost function is the mean sum of squared differences between consecutive concentration-distance curves. The velocity $v(t)$ in millimeters per second for shift $S$ is given by

$$
v(t)=s \times F R \text { where } F R \text { is the frame rate in frames per second } .
$$


The original concentration-curve matching algorithm (ORG algorithm) selects the value of $s$ for which $\boldsymbol{\Psi}(s, t)$ is minimum. This value, $s_{o p t}$, is the value for which there is the best match between consecutive concentration-distance curves. The estimated contrast medium velocity is then given by

$$
v(t)=s_{o p t} \times F R .
$$

In order to make this algorithm less susceptible to noise in the angiographic data, polynomials can be fitted to the concentration-distance curves prior to carrying out the curve matching procedure. This is termed the polynomial approximation algorithm (PA algorithm). The order of the polynomial used is dependent on the length of the vessel segment. For this study, fourth order polynomials were found to model the shape of the concentration-distance curves without modeling the noise component.

Instead of choosing the value of the shift that produces the minimum value of the cost function $\boldsymbol{\Psi}$, the model-based algorithm (MB algorithm) constrains the value of the shift chosen by using the waveform shape information. Let the volumetric flow waveform be $\mathbf{G}(t)$ where $t$ is the time measured in percentage cardiac cycle and volumetric flow is measured in millilitres per minute. Using the waveform shape model, $\mathbf{G}(t)$ is estimated by

$$
\mathbf{G}(t)=\overline{\mathbf{P}}+\sum_{i=1}^{i=S} w_{i} \mathbf{e}_{i} \text { where } S \text { is the total number of eigenvectors } .
$$

The model fitting task entails selecting the values of $w_{i}$ that minimize the mean value of the cost function $\boldsymbol{\Psi}(s, t)$.

Step 1:

The concentration-distance curve function $\mathbf{C}(x, t)$ will contain one or more cardiac cycles depending on the cardiac rate and the number of frames acquired. The cycle for which the shape model is to be fitted is identified using the velocity waveform derived from the original concentration-curve matching algorithm. $t_{s}$ is identified as the starting frame for the cycle and $t_{e}$ is identified as the end frame of the cycle. The cycle length is then given by

$$
T_{c}=t_{e}-t_{s}+1
$$

The waveform $\mathbf{G}(t)$ is rescaled in the time axis so that $0 \leq t \leq T_{c}-1$.

Step 2:

The flow waveform $\mathbf{G}(t)$ is converted into units of mm shift per frame by

$$
\mathbf{s}(t)=\frac{\mathbf{G}(t)}{k \times A \times F R} \text { where } A \text { is the vessel cross-sectional area }
$$

and $k=6$ is the unit conversion factor . 
Step 3:

The cost $\Phi$ is calculated by

$$
\Phi=\frac{1}{T_{c}} \sum_{t=t_{s}}^{t=t_{e}} \Psi\left(s\left(t-t_{s}\right), t\right) .
$$

The values of the weighting factors $w_{i}$ are found which minimize the cost $\Phi$. This is done by the downhill simplex method. Only 10 eigenvectors were used for the generation of the fitted waveform.

\section{Results}

Figure 8 shows the model-based $\mathrm{x}$-ray computed flow values and the EMF recording for 1 of the 10 experiments. The waveform produced by the MB algorithm follows the EMF waveform closely and is less noisy than the waveforms produced by the ORG and PA x-ray algorithms.

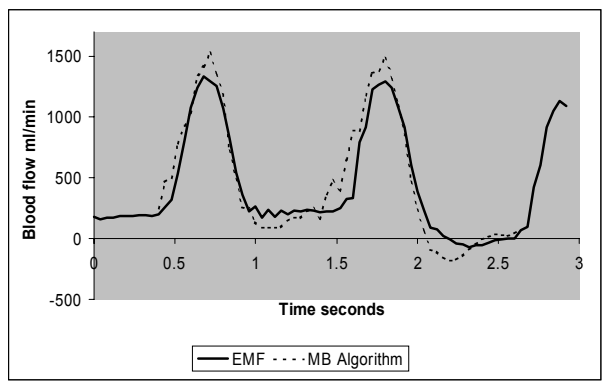

Fig. 8. An example of blood flow waveform produced by the model-based x-ray algorithm and the corresponding waveform from the electromagnetic flow meter.

Figure 9 and figure 10 show scatter plots for instantaneous flow values and average flow rates produced by the MB algorithm. Table 1 summarizes the results obtained using all three algorithms for instantaneous and average flow comparison with the EMF. The correlation between instantaneous and average flow rates between the $\mathrm{x}$ ray measurements and the EMF was highly significant for all three algorithms. Each algorithm produced a mean over-estimation of both instantaneous and average flow but the limits of agreement ${ }^{(10)}$ as expressed by the $95 \%$ confidence interval for the difference between $\mathrm{x}$-ray and EMF flow values were narrower for the model-based algorithm. 


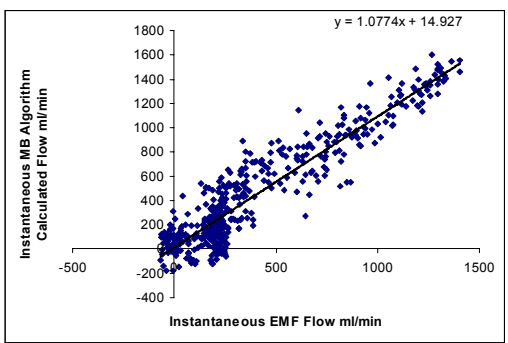

Fig. 9. Scatter plot of instantaneous flow values calculated using the model-based algorithm and the electromagnetic flow meter.

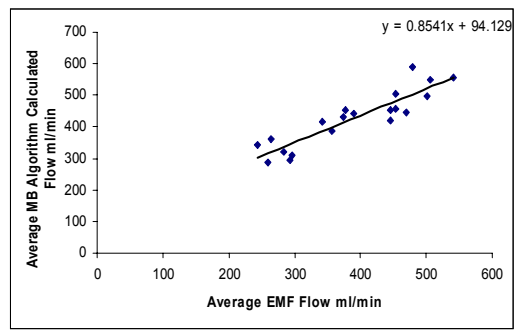

Fig. 10. Scatter plot of average flow values calculated by the model-based algorithm and the electromagnetic flow meter.

\begin{tabular}{|c|c|c|c|}
\hline & MB Algorithm & ORG Algorithm & PA Algorithm \\
\hline Instantaneous Flow Correlation & 0.934 & 0.908 & 0.913 \\
\hline Mean Difference In Instantaneous Flow & 44.4 & 17.1 & 29.1 \\
And 95\% CI ml/min ( X-Ray - EMF) & -266.0 to 354.7 & -346.8 to 380.9 & -314.0 to 372.1 \\
\hline Average Flow Correlation & 0.906 & 0.913 & 0.909 \\
\hline Mean Difference In Average Flow & 37.4 & 24.2 & 35.1 \\
And 95\% CI ml/min ( X-Ray - EMF) & -41.5 to 116.3 & -70.2 to 118.7 & -53.8 to 123.9 \\
\hline
\end{tabular}

Table 1. Summary of results of instantaneous and average flow measurement by the x-ray techniques compared to the electromagnetic flow meter measurments. $\mathrm{p}<0.001$ for all correlation coefficients.

In order to study the effect of image quality on the performance of the algorithms, different amounts of Gaussian-distributed noise were added to one of the parametric images obtained. Gaussian noise with standard deviation of $1 \%$ to $30 \%$ of the maximum pixel grey value in the parametric image was used. Figure 11 illustrates the correlation with the EMF of instantaneous flow values obtained by the three algorithms as function of added percentage noise. It can be seen that there is minimum reduction of correlation for the $\mathrm{MB}$ algorithm and maximum for the ORG algorithm, with the PA algorithm performing marginally better than ORG algorithm.

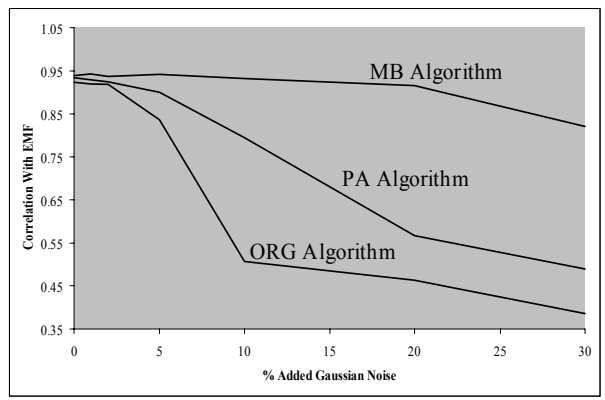

Fig. 11. Variation of correlation between $x$-ray and electromagnetic flow meter instantaneous values as a function of percentage added Gaussian noise. 


\section{Conclusion}

We have demonstrated a novel model-based algorithm to measure blood flow from dynamic digital x-ray images of arteries. The model-based algorithm has shown narrower limits of agreement with the electromagnetic flow meter measurements when compared to our existing algorithms for both instantaneous and average flow values. Also, this algorithm has been shown to be less sensitive to degradation in image quality than the other algorithms. Compared to the images of our vessel phantom, clinical angiograms will be of poorer image quality. Factors such as scatter, beam hardening, over-lapping vessels, and patient motion will contribute to reduce image quality. The model-based algorithm is therefore likely to give more reliable estimates of blood flow from clinical data than our previously reported techniques. We a view to test this algorithm clinically, we are collecting blood flow waveforms from target arteries in healthy volunteers using Doppler ultrasound. These waveforms will be used to form waveform shape models for these different arteries.

\section{References}

1. Shpilfoygel S. D., Close R. A., Valentino D. J., and Duckwiler G. R., X-ray Videodensitometric Methods for Blood Flow and Velocity Measurement: A Critical Review of Literature. Med.Phys. 27[9], 2008-2023. 2000.

2. Evans D.H., Archer L.N.J., and Levene M.I, The Detection of Abnormal Neonatal Cerebral Haemodynamics using Principal Component Analysis of the Doppler Ultrasound Waveform. Ultrasound Med Biol 11, 441-449. 1985.

3. MacPherson D.S., Evans D.H., and Bell P.R.F., Common Femoral Artery Doppler Waveforms: A Comparison of Three Methods of Objective Analysis with Direct Pressure Measurements. Br J Surg 71, 46-49. 1984.

4. Evans D. H., The Interpretation of Continuous Wave Ultrasonic Doppler Blood Velocity Signals Viewed as a Problem in Pattern Recognition. J.Biomed.Eng 6[4], 272-280. 1984.

5. Prytherch D. R., Evans D. H., Smith M. J., and Macpherson D. S., On-line Classification of Arterial Stenosis Severity using Principal Component Analysis Applied to Doppler Ultrasound Signals. Clin.Phys.Physiol Meas. 3[3], 191-200. 1982.

6. Evans D. H., Macpherson D. S., Bentley S., Asher M. J., and Bell P. R., The Effect of Proximal Stenosis on Doppler Waveforms: A Comparison of Three Methods of Waveform Analysis in an Animal Model. Clin.Phys.Physiol Meas. 2[1], 17-25. 1981.

7. Martin T.R.P., Barber D.C., Sherriff S.B, and Prichard D.R., Objective Feature Extraction Applied to the Diagnosis of Carotid Artery Disease: A Comparative Study with Angiography. Clin Phys Physiol Meas 1, 71-81. 1980.

8. Seifalian A.M., Hawkes D.J., Colchester A.C.F., and Hobbs K.E.F., A New Algorithm for Deriving Pulsatile Blood Flow Waveforms Tested using Simulated Dynamic Angiographic Data. Neuroradiology 13, 263-269. 1989.

9. Seifalian A.M., Hawkes D.J., Bladin C., Colchester A.C.F., and Hobbs K.E.F., Blood Flow Measurements Using 3D Distance-Concentration Functions Derived From Digital X-Ray Angiograms. Cardiovascular Imaging, 425-442. 1996. Netherlands, Kluwer Academic.

10. Bland J.M. and Altman D.G., Statistical Methods for Assessing Agreement Between Two Methods of Clinical Measurement. The Lancet 1, 307-310. 1986. 